\title{
Efecto de la formación del manto de lodos sobre el compor- tamiento hidrodinámico de un reactor UASB a escala real. estudio de caso: Sotaquirá, Colombia
}

Effect of the sludge blanket formation on the hydrodynamic behavior of a full scale UASB-reactor, a case study of Sotaquirá, Colombia

\author{
ANDREA PÉREZ-VIDAL \\ Ingeniera Sanitaria \\ Doctora en Ingeniería con énfasis en Ingeniería Sanitaria y Ambiental. \\ Universidad Santiago de Cali, Colombia \\ andrea.perez00@usc.edu.co \\ JAIME DÍAZ-GÓMEZ \\ Ingeniero Sanitario \\ Magister en Environmental Science and Technology \\ Grupo de Investigación Gestión del Recurso Hídrico \\ Universidad de Boyacá, Colombia \\ jaimediaz@uniboyaca.edu.co
}

\author{
DIEGO CAMILO-GUÍO \\ Ingeniero Sanitario-Ingeniero Ambiental \\ Grupo de Investigación Gestión del Recurso Hídrico \\ Universidad de Boyacá, Colombia \\ dieguio@uniboyaca.edu.co
}

\section{VANESSA CASTAÑEDA-MORENO}

Ingeniera Sanitaria-Ingeniera Ambiental

Corporación Autónoma Regional de Boyacá

(CORPOBOYACA)

vcastaneda@corpoboyaca.gov.co 


\section{RESUMEN}

La evaluación del patrón de flujo en reactores biológicos empleando trazadores es un método ampliamente reconocido para identificar posibles deficiencias durante su operación. En este estudio se empleó Rodamina WT como trazador para identificar los cambios en el patrón de flujo causados por el crecimiento del manto de lodos en un reactor UASB de $32 \mathrm{~m}^{3}$ que trata aguas residuales domésticas. La evaluación se realizó al comienzo de la operación del reactor y al final del periodo de arranque. El patrón de flujo se identificó empleando los modelos de dispersión axial, tanques en serie y Wolf - Resnick. La evaluación hidrodinámica inicial mostró predominio de mezcla completa $(76.6 \%)$ en el reactor y un índice de dispersión de 0.20 , valores que disminuyeron al final del arranque cuando se conformó el manto de lodos (59.2\% mezcla completa, $40.8 \%$ flujo pistón y 0.14 índice de dispersión). Se evidenció presencia de cortos circuitos y ausencia de zonas muertas en las dos condiciones evaluadas. Aunque la formación del manto de lodos influyó en el comportamiento hidrodinámico en el reactor, la variación del Tiempo de Retención Hidráulico se mostró como un factor clave en el patrón de flujo predominante en el reactor.

Palabras clave: Ensayo de trazadores, flujo pistón, régimen hidráulico, Índice de dispersión, flujo de mezcla completa.

\section{ABSTRACT:}

The use of tracers in the evaluation of the flow pattern in biological reactors is a widely recognized method for identifying possible deficiencies during their operation. In this study, Rhodamine WT was used as a tracer to identify changes in the flow pattern caused by the growth of the sludge blanket in a $32 \mathrm{~m} 3$ UASB reactor treating domestic wastewater. The evaluation was conducted at the beginning of reactor operation and at the end of the start-up period. The flow pattern was identified using the axial dispersion, continuous stirred-tank reactor and Wolf - Resnick models. The initial hydrodynamic evaluation, showed predominance of completely mixed flow (76.6\%) and a dispersion index of 0.20 . These values decreased at the end of start-up period when the sludge blanket was formed $(59.2 \%$ mixed flow, $40.8 \%$ plug flow and a dispersion index of 0.14 ). In both conditions, there was presence of short circuits, but absence of dead zones. Although the formation of sludge blanket influenced the hydrodynamic behavior, the variation of hydraulic retention time was found to be a key factor to define the predominant flow pattern in the reactor.

Keywords: Completely mixed flow, dispersion index, hydraulic regime, plug flow, tracer studies.

Pérez, A., Díaz, J., Guío, C. \& Castañeda, V. (2014, Noviembre 14). Efecto de la formación del manto de lodos sobre el comportamiento hidrodinámico de un reactor UASB a escala real. 


\section{INTRODUCCIÓN}

En el tratamiento de las aguas residuales se puede encontrar los tres estados de la materia (sólido, líquido y gas) y su interacción está relacionada directamente con procesos que se pueden optimizar si se proporcionan las condiciones hidrodinámicas adecuadas (Giácoman et al, 2006). El modelo hidráulico de un reactor es una función del patrón de flujo y el régimen de mezcla en la unidad, los cuales a su vez dependen de la geometría del reactor, la cantidad de energía introducida por unidad de volumen, y el tamaño o cambio de la escala. En términos del flujo, los reactores pueden ser de flujo intermitente o flujo continuo (von Sperling, 1996). Los reactores de flujo continuo pueden a su vez clasificarse en flujo ideal, pistón o mezcla completa y flujo no ideal (CEPIS, 2004; Levenspiel, 1997).

El Tiempo de Retención Hidráulico (TRH) indica el tiempo teórico que los elementos de un fluido permanecerían en el reactor. Este tiempo se diferencia del tiempo de retención real de todos los elementos del fluido el cual puede ser diferente dependiendo de las rutas de flujo que estos sigan y las características de mezcla del reactor (Grady et al, 2011).

Por lo general en el diseño de reactores biológicos o químicos solo se consideran los balances de masa y la cinética de las reacciones sin tener en cuenta los fenómenos de transferencia de masa y energía o el patrón de mezcla, asumiendo de esta forma que el reactor opera bajo condiciones ideales (Morgan- Sagastume, Jiménez y Noyola, 1997). Aunque algunos reactores diseñados de esta forma pueden ser operados con buenos resultados, otros no logran alcanzar el rendimiento esperado debido a limitaciones en el transporte del sustrato, alta influencia de la temperatura en la velocidad de la reacción, así como por la presencia de imperfecciones en el patrón de flujo como cortos circuitos y zonas muertas (Lema et al, 1991).

Para identificar el patrón predominante de flujo y la magnitud de las zonas con imperfecciones, pueden emplearse diferentes modelos matemáticos basados en los resultados obtenidos con el método experimental estimulo-respuesta, en el que se inyecta un trazador en el afluente y se obtiene como respuesta una concentración del trazador en el efluente distribuida en el tiempo (Levenspiel, 1997; Grady et al, 2011). Esta técnica permite el análisis de la distribución de los tiempos de residencia, el cual es un método útil para describir el patrón de flujo y las características de mezcla, lo que a su vez puede ser usado para estimar el rendimiento de los procesos de remoción que se llevan a cabo dentro de un reactor (Méndez et al., 2011). 
La aplicación del trazador puede hacerse en forma instantánea o continua, siendo la primera la más usada. Entre las sustancias trazadoras pueden citarse colorantes como fluoresceína o rodamina; iones como Cloruros especialmente de Sodio, Potasio o Litio; Fluoruros o Nitratos; elementos radiactivos como isótopos; ácidos Clorhídrico o Benzóico y otras sustancias químicas como Alizarim, Sapirol y Naptol (Jiménez, Noyola y Capdeville, 1998). Para reactores biológicos anaerobios es recomendable que en la selección del trazador se consideren criterios como: no biodegradable para evitar adsorción en la biomasa; no absorción en el biogás; estable en el tiempo y bajo condiciones del proceso ( $\mathrm{pH}$ y potencial rédox); soluble en el agua y preferiblemente con elevado peso molecular para evitar problemas por difusión; rango de concentración con respuesta lineal y buena sensibilidad (Rocha et al, 2000) y no tóxico para la biomasa en las concentraciones empleadas.

El Reactor Anaerobio de Flujo Ascendente y Manto de Lodos, conocido comúnmente como UASB (Upflow Anaerobic Sludge Blanket Reactor) fue desarrollado en la década de los 70's - 80's por el Profesor Gatze Lettinga de la Universidad de Wageningen (Lettinga et al, 1980) y su comportamiento hidráulico se puede describir por medio de una combinación de patrones de flujo ideal. De acuerdo con Hulshoff y Lettinga (1988) citado por Arroyave et al. (2005), en el fondo del reactor el flujo es predominantemente de tipo pistón. En el manto de lodo, por la presencia del biogas, el comportamiento es turbulento y el flujo tiende a ser completamente mezclado, y el sedimentador, el flujo es laminar tendiente a flujo a pistón. Bolle et al (1986), empleó un modelo para el flujo del fluido en un reactor UASB el cual se divide en tres zonas: el lecho de lodos y el manto de lodos caracterizados como reactores de mezcla completa y la zona de clarificación como un reactor de flujo a pistón (ver Figura 1).

Según Arroyave et al (2005), una buena operación de estos reactores se puede acompañar con una evaluación hidráulica del sistema, durante y después de la fase de arranque. Esto se hace con el fin de identificar el patrón de flujo predominante y la magnitud de las zonas con imperfecciones, buscando su optimización y la solución de problemas relacionados con su eficiencia.

Estudios realizados por Zeng et al. (2005), quienes evaluaron el modelo de dispersión axial en un reactor UASB, evidenciaron que el manto de lodos influenciaba directamente en el aumento del coeficiente de dispersión, el cual, disminuía proporcionalmente con la altura del reactor, alcanzando los valores más bajos de dispersión a partir del límite superior del manto de lodo y permaneciendo similar en la zona se sedimentación. Por otra parte, van Haandel y Lettinga (1994), evaluaron el patrón de flujo un reactor UASB piloto de $64 \mathrm{~m}^{3}$ alimentado con agua residual doméstica, empleando Litio como trazador, encontrando que tanto al comienzo del arranque como después de un mes de operación, el patrón predominante de flujo es mezcla completa con una reducción de la fracción de zonas muertas desde 0.15 hasta 0.08 . Peña et al. (2006) también encontraron predominancia de mezcla completa con zonas muertas localizadas principalmente en el lecho y el manto de lodos, y cortos circuitos. 


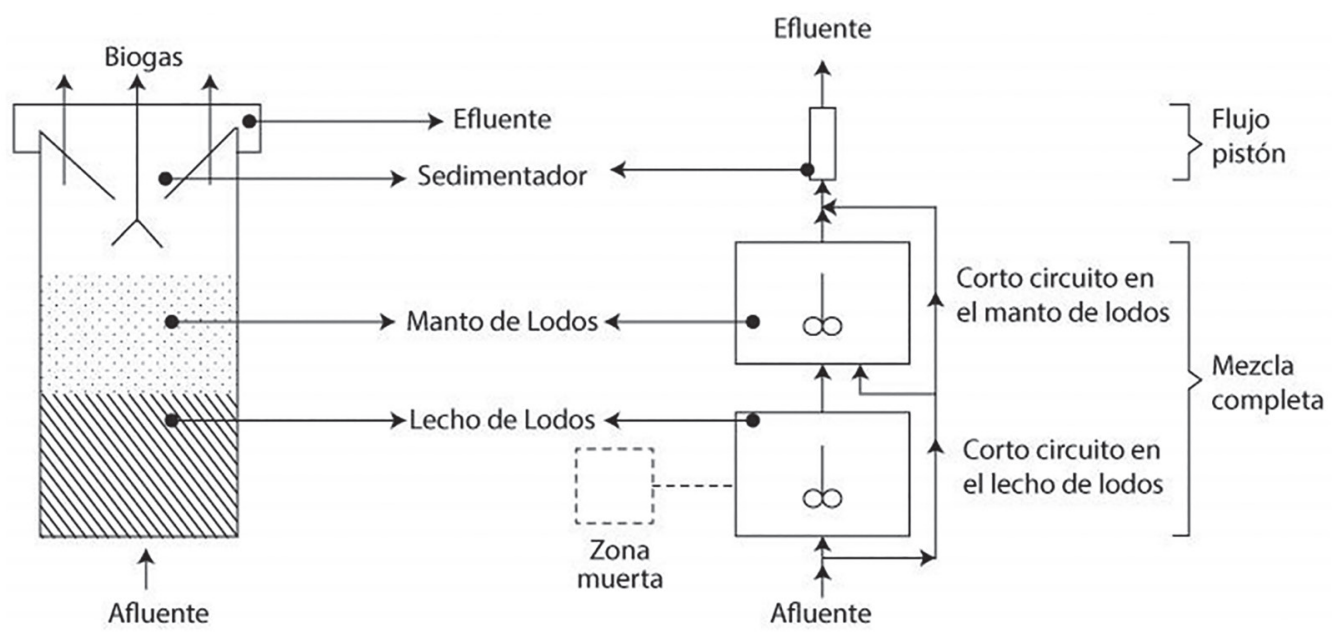

Figura 1. Modelo del patrón de flujo en un Reactor UASB

Fuente: Bolle et al. (1986)

Los resultados de Zeng et al. (2005) y Peña et al. (2006), coinciden en que los eventos de sobrecarga hidráulica del reactor definen un patrón de flujo no ideal caracterizado por un aumento en la dispersión. Lo anterior ratifica que la velocidad de flujo ascendente es uno de los principales factores que afecta la eficiencia de remoción de materia orgánica de este tipo de reactores (Íñiguez y Camacho, 2011).

En este estudio se evaluó el comportamiento hidrodinámico del reactor UASB de la Planta de Tratamiento de Aguas Residuales (PTAR) del municipio de Sotaquirá (Boyacá) mediante dos ensayos de trazadores. El primero se llevó a cabo al inicio del arranque del reactor sin manto de lodos y el segundo a los 6 meses de su entrada en operación. Los resultados del estudio permitieron evaluar la influencia del manto de lodos sobre el patrón de flujo del reactor.

\section{METODOLOGÍA}

El municipio de Sotaquirá está localizado en el Departamento de Boyacá, en el centro-oriente de Colombia, en la región del Alto Chicamocha. La altura sobre el nivel del mar es de 2800 metros. De acuerdo al censo poblacional realizado por el Departamento Administrativo Nacional de Estadística (DANE) en el año 2005, la población en la cabecera municipal es de 721 Habitantes. 
La Planta de Tratamiento de Aguas Residuales del municipio recibe las aguas residuales transportadas por un alcantarillado combinado y opera con un caudal promedio de $1.5 \mathrm{~L} / \mathrm{s}$. Cuenta con tres líneas de tratamiento en paralelo, cada una operando con un 1/3 del caudal total. El estudio se enfocó en la línea en la cual opera un Reactor UASB (volumen de $23 \mathrm{~m}^{3}$ ) con un caudal promedio de $0.5 \mathrm{~L} / \mathrm{s}$ y un Tiempo de Retención Hidráulico de diseño de 8 horas. La temperatura promedio de las aguas residuales es de $18^{\circ} \mathrm{C}$ y la densidad de puntos de entrada en el reactor es de $1.8 \mathrm{~m}^{2} /$ punto. La Figura 2 muestra un esquema de las dimensiones y configuración del reactor.
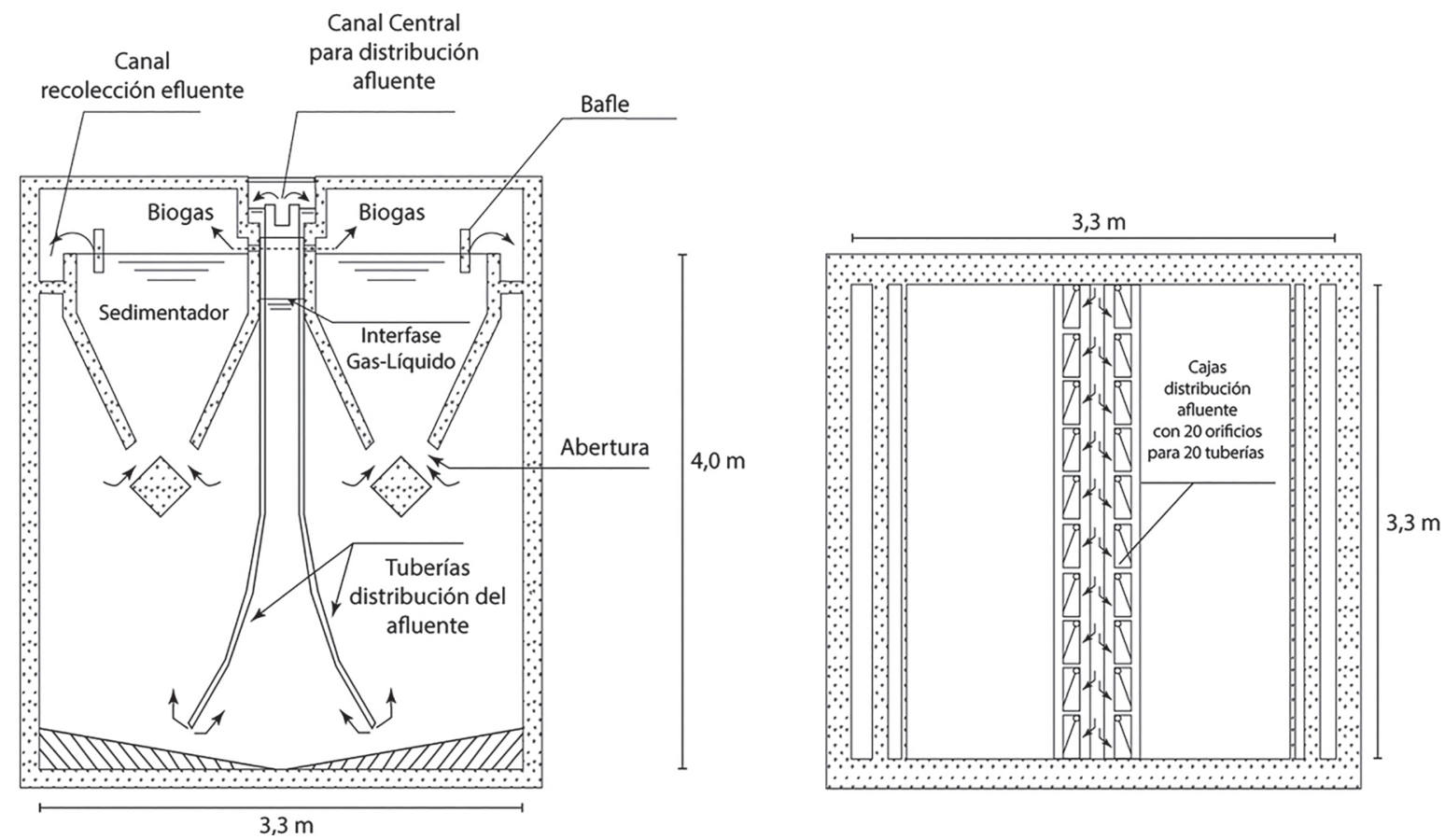

Figura 2. Esquema del reactor UASB de la PTAR de Sotaquirá (Boyacá) Fuente: Adaptado de Chernicharo (1997)

El desarrollo experimental del estudio se dividió en dos fases: i) Arranque del reactor (sin inóculo) y ii) Finalización del arranque. En cada una de las fases se realizó un ensayo de trazadores con aplicación de una dosis instantánea de Rodamina WT al 20\% (Turner Design, PID 70301027) con el fin de evaluar el comportamiento hidrodinámico del reactor frente al proceso de formación del manto de lodos. De forma paralela, se realizó el seguimiento de las eficiencias de remoción de DQO y SST en cada fase. La Tabla 1 describe las características que acompañaron cada fase del estudio. 


\begin{tabular}{|l|l|}
\hline \multicolumn{1}{|c|}{ FASE } & \multicolumn{1}{c|}{ CARACTERÍSTICAS OPERACIONALES } \\
\hline Arranque del reactor & $\begin{array}{l}\text { Al inicio de la puesta en marcha de la PTAR entraron en funcionamiento solo } \\
\text { dos líneas de tratamiento, por lo que el arranque del reactor UASB se inició } \\
\text { con un caudal promedio de 0.8 L/s. No se empleó ningún inóculo siguiendo lo } \\
\text { reportado en algunas experiencias relacionadas con el arranque de reactores } \\
\text { UASB tratando aguas residuales domesticas (Schellinkhout y Collazos, 1992; } \\
\text { Álvarez et al, 2006). Se resalta que este primer ensayo de trazadores se reali- } \\
\text { zó bajo una condición climática seca. }\end{array}$ \\
\hline Finalización del \\
arranque & $\begin{array}{l}\text { El arranque del reactor UASB tuvo una duración de 6 meses, periodo para el } \\
\text { cual se alcanzó el crecimiento de los microorganismos que conformaron el } \\
\text { manto de lodos, lográndose la eficiencia de remoción de DQO esperada de } \\
\text { 60\%. Para esta fase la PTAR ya operaba con las tres líneas de tratamiento, por } \\
\text { lo que el caudal promedio de operación del reactor fue de 0.5 L/s. Una vez } \\
\text { finalizado este periodo se realizó el segundo ensayo de trazadores, el cual } \\
\text { también estuvo acompañado de una condición climática seca. }\end{array}$ \\
\hline
\end{tabular}

Tabla1. Descripción de las fases metodológicas del estudio.

La dosis instantánea de Rodamina WT fue de $10 \mathrm{ml}$ de la solución al 20\% disuelta en 7 L de agua destilada y fue aplicada en el canal de entrada del reactor UASB. La medición de la concentración de trazador a la salida del reactor se realizó utilizando un Fluorómetro Portátil Aquafluor ${ }^{\mathrm{TM}}$ el cual fue previamente calibrado con una solución patrón de 100 ppb.

Durante los dos ensayos de trazadores se monitoreó el caudal de entrada al reactor. La variación horaria se debió exclusivamente a las descargas domésticas del municipio. El TRH teórico del Reactor UASB, en ambos ensayos, varió en función del caudal alcanzándose un valor promedio de 7.1 y 15.6 horas respectivamente. Siguiendo lo recomendado por CEPIS (2004) y Galvis (2005) los ensayos se programaron para una duración equivalente a 3 veces el TRH teórico, sin embargo, tan pronto se alcanzaron concentraciones consecutivas de trazador de $0.00 \mathrm{ppb}$, se suspendió el ensayo. De acuerdo con lo anterior el primer ensayo tuvo una duración de 24 horas y el segundo de 28 horas.

Los resultados de los ensayos de trazadores fueron inicialmente analizados a partir de la curva de tendencia de la concentración del trazador. Posteriormente se construyó la curva de distribución de tiempos E(t) para determinar el TRH real o experimental, siguiendo lo establecido por CEPIS (2004) y Weber \& DiGiano (1996). Adicionalmente, se aplicaron los modelos matemáticos de Wolf-Resnick (1963), dispersión axial y reactores completamente mezclados en serie (Levenspiel, 1997). A continuación se describe brevemente el soporte matemático de cada uno de los modelos usados. 


\section{Modelo de Wolf-Resnick}

El modelo empírico (de caja negra) propuesto por David Wolf y William Resnick (1963) permite determinar de manera objetiva y sencilla el porcentaje del flujo que presenta una característica de pistón (Ecuación 1), espacios muertos en el sistema (Ecuación 2) y el porcentaje de zona de mezcla completa (Ecuación 3) (CEPIS, 2004). Los valores de $\theta$ y $\alpha$ se calculan a partir de la curva presentada en la Figura 3.

$$
p=\frac{\theta \tan \alpha}{0.435+\theta \tan \alpha}
$$

$$
m=1-\frac{\theta}{p}
$$

$$
M=1-p
$$

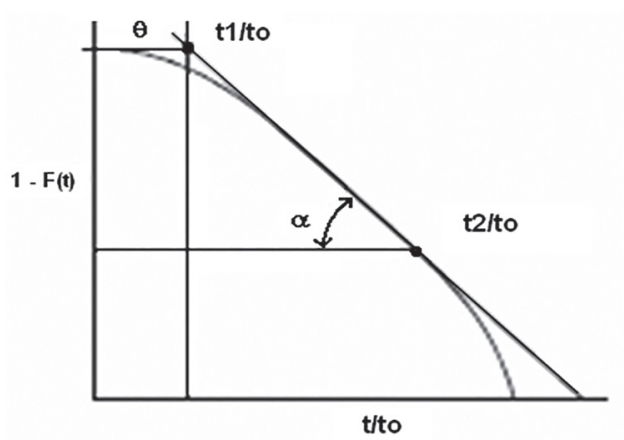

Figura 3. Curva de 1-F(t). Cantidad de trazador que permanece en el reactor Fuente: CEPIS (2004). 


\section{Modelo de dispersión axial}

El parámetro que caracteriza a este modelo es el coeficiente $D\left(\mathrm{~m}^{2} / \mathrm{s}\right)$, el cual expresa tanto la mezcla interna como la difusión molecular. El término adimensional (D/uL), denominado número de dispersión del reactor, es el parámetro que mide el grado de dispersión axial, donde u es la velocidad del flujo y $\mathrm{L}$ la longitud o recorrido del fluido desde el punto de entrada hasta el punto de salida. Para el cálculo del término $\mathrm{D}$ o D/uL se debe estudiar la forma de la curva del trazador a medida que sale del reactor y calcular el tiempo medio de residencia $\left(\mathrm{t}_{\mathrm{m}}\right)$ y la varianza de la curva $\left(\sigma^{2}\right)$. El cálculo de la varianza se realiza con la Ecuación 4.

$$
\sigma^{2}=\frac{\sum t_{i}^{2} * C_{i}}{\sum C_{i}}-t_{m}^{2}=\frac{\sum t_{i}^{2 *} C_{i}}{\sum C_{i}}-\left(\frac{\Sigma t_{i} C_{i}}{\Sigma C_{i}}\right)^{2}
$$

Donde:

$\mathrm{t}_{\mathrm{i}}=$ Tiempo inicial de salida del trazador $(\mathrm{h})$

$\mathrm{t}_{\mathrm{m}}=$ Tiempo teórico de residencia $(\mathrm{h})$

$\mathrm{C}_{\mathrm{i}}=$ Concentración del trazador al tiempo $\mathrm{t}(\mathrm{mg} / \mathrm{l})$

$\mathrm{C}_{\mathrm{o}}=$ Concentración inicial del trazador $(\mathrm{mg} / \mathrm{l})$

Para grados de dispersión pequeños (D/uL es pequeño) la curva del trazador no cambia significativamente de forma a su paso por el punto de medida y el número de dispersión axial puede ser estimado por la siguiente ecuación.

$$
\sigma_{\theta}^{2}=\frac{\sigma^{2}}{-2}=2\left(\frac{\mathrm{D}}{\mathrm{t}}\right)
$$

Donde:

$$
\begin{aligned}
& \theta=\text { Varianza }\left(\mathrm{h}^{2}\right) \\
& \overline{\mathrm{t}}=\text { Tiempo de residencia real }(\mathrm{h})
\end{aligned}
$$


Para dispersiones grandes $(\mathrm{D} / \mathrm{uL}>0.01)$, con tendencia a flujo mezclado la curva del trazador cambia significativamente de forma, durante el tiempo que pasa por el punto de medida, la curva obtenida es asimétrica y presenta una ligera cola. Para reactores abiertos, la varianza puede ser determinada con la Ecuación 6:

$$
\sigma_{\theta}^{2}=\frac{\sigma^{2}}{-2}=2\left(\frac{\mathrm{D}}{\mathrm{t}}\right)+8\left(\frac{\mathrm{D}}{\mathrm{uL}}\right)^{2}
$$

\section{Modelo de reactores completamente mezclados en serie}

El modelo puede expresarse de acuerdo con la ecuación 7 para "n" reactores igual a "N" (Levenspiel, 1997). El valor de $\mathrm{N}$ puede calcularse a partir de la distancia máxima entre los puntos de inflexión o la varianza de la curva $\mathrm{C}$. La curva $\mathrm{E}(\mathrm{t})$ o RTD se va haciendo más asimétrica a medida que aumenta $\mathrm{N}$.

$$
E(t)=\frac{N^{*}\left(N^{*} \theta\right)^{N-1}}{(N-1) !} * e^{-N^{*} \theta}
$$

Donde:

$\theta=\mathrm{t} / \mathrm{t}_{\mathrm{R}}(-)$

$t_{R}=$ tiempo medio de residencia en el conjunto de $N$ tanques $\left(N^{*} t_{i}\right)(h)$

$t_{i}=$ tiempo medio de residencia en cada tanque (h)

\section{RESULTADOS Y DISCUSIÓN}

\section{Análisis de la curva de tendencia de la concentración de trazador}

Generalmente, la forma que toma la curva de concentración da una primera indicación del comportamiento hidráulico y tipo de flujo predominante en el reactor (Galvis, 1990). En la Figura 3 se muestran las curvas de tendencia obtenidas en los ensayos de trazadores para las dos fases del estudio. La Tabla 2 sintetiza el cálculo de los parámetros recomendados por CEPIS (2004) para el análisis de la curva de tendencia de la concentración del trazador. De acuerdo con CEPIS (1992), el tiempo que transcurre desde la inyección del trazador hasta que aparece en el efluente $\left(\mathrm{t}_{\mathrm{i}}\right)$ indica la fracción de flujo a pistón, lo que permite evidenciar que el reactor en la Fase 1 presentó una mayor fracción de flujo a pistón (p) si se compara con la Fase 2; sin embargo, se destaca que la proporción de mezcla completa (M) es predominantemente mayor en la Fase 1. Probablemente la ausencia del manto de lodos en la Fase 1 
favoreció un mayor valor de "t. al presentar un comportamiento de flujo similar al presentado en la zona de clarificación, el cual, como lo indica Bolle et al., (1986), tiende a tener un patrón de flujo predominante de tipo pistón.
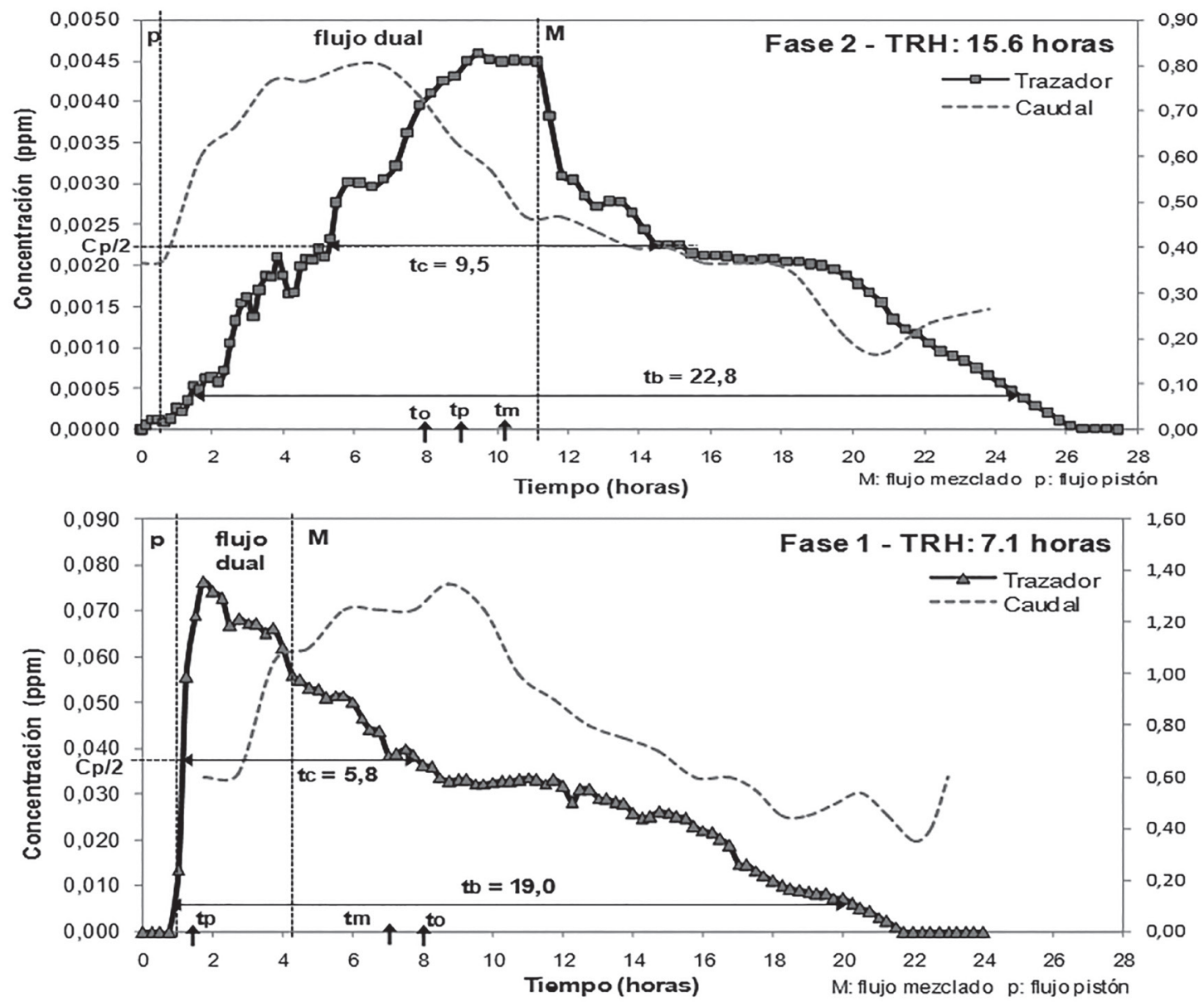

Figura 3. Curvas de concentración de trazador vs tiempo y variación del caudal. 


\begin{tabular}{|c|c|c|c|}
\hline RELACIÓN & FASE 1 & FASE 2 & INTERPRETACIÓN* \\
\hline$t_{i} / t_{0}=$ & 0.14 & 0.01 & $\begin{array}{l}t_{i} / t_{o}=0 \text { (flujo mezcla completa) } \\
t_{i} / t_{o}=1 \text { (flujo pistón) } \\
t_{i} / t_{0}<0,3 \text { (cortos circuitos - paso directo entre la entrada y salida } \\
\text { del reactor) }\end{array}$ \\
\hline $\mathrm{t}_{\mathrm{m}} / \mathrm{t}_{\mathrm{o}}=$ & 0.94 & 0.65 & $\begin{array}{l}\mathrm{t}_{\mathrm{m}} / \mathrm{t}_{\mathrm{o}}<1 \text { (cortos circuitos) } \\
\mathrm{t}_{\mathrm{m}} / \mathrm{t}_{\mathrm{o}}>1 \text { (acumulación indeseada de trazador }- \text { zona muerta o } \\
\text { error de ensayo) }\end{array}$ \\
\hline$t_{p} / t_{o}=$ & 0.25 & 0.59 & $\begin{array}{l}t_{p} / t_{o}=0 \text { (existe solo mezcla completa) } \\
t_{p} / t_{0}=1 \text { (existe solo flujo pistón) }\end{array}$ \\
\hline$t_{c} / t_{o}=$ & 0.81 & 0.61 & $\begin{array}{l}\mathrm{t}_{c} / \mathrm{t}_{\mathrm{o}} \approx 0,7 \text { (relacionado con corrientes de inercia o turbulencia - } \\
\text { predominio de mezcla completa) }\end{array}$ \\
\hline$t_{b} / t_{o}$ & 2.68 & 1.46 & $\begin{array}{l}\mathrm{t}_{\mathrm{b}} / \mathrm{t}_{\mathrm{o}} \approx 2,3 \text { (relacionado con corrientes de inercia o turbulencia - } \\
\text { predominio mezcla completa) }\end{array}$ \\
\hline$e=$ & 2.72 & 0.63 & $\begin{array}{l}\text { e } \geq 2,3 \text { (flujo mezcla completa) } \\
\text { e }=0 \text { (flujo pistón) }\end{array}$ \\
\hline \multicolumn{3}{|c|}{$\begin{array}{l}\mathrm{C}_{\mathrm{p}}=\text { concentración máxima a la salida }=0.07638 \mathrm{ppm} \text { y } 0,004492 \mathrm{ppm} \text { para cada fase } \\
\text { respectivamente. }\end{array}$} & $\begin{array}{l}\text { oras para cada fase respectivamente } \\
\text { salida }=0.07638 \mathrm{ppm} \text { y } 0,004492 \mathrm{ppm} \text { para cada fase }\end{array}$ \\
\hline \multicolumn{4}{|c|}{$\begin{array}{l}t_{p}=\text { tiempo modal, correspondiente a la presentación de la máxima concentración }=1.75 \text { h y } 9.2 \\
\text { h para cada fase respectivamente. }\end{array}$} \\
\hline \multicolumn{4}{|c|}{$\begin{array}{l}t_{i}=\text { tiempo inicial desde que se aplica el trazador hasta que aparece en el efluente }=1.0 \text { h y } 0.17 \\
\text { h para cada fase respectivamente. }\end{array}$} \\
\hline \multicolumn{4}{|c|}{$\begin{array}{l}\mathrm{t}_{\mathrm{c}}=\text { tiempo en que la concentración es mayor que } \mathrm{C}_{\mathrm{p}} / 2=5.8 \text { h y } 9.5 \text { h para cada fase } \\
\text { respectivamente. }\end{array}$} \\
\hline \multicolumn{4}{|c|}{$\begin{array}{l}\mathrm{t}_{\mathrm{b}}=\text { tiempo en que la concentración es mayor que } \mathrm{C}_{\mathrm{p}} / 10=19.0 \text { h y } 22.8 \mathrm{~h} \text { para cada fase } \\
\text { respectivamente. }\end{array}$} \\
\hline
\end{tabular}




\begin{tabular}{|l|l|l|l|}
\hline RELACIÓN & FASE 1 & FASE 2 & INTERPRETACIÓN* \\
\hline $\mathrm{t}_{\mathrm{m}}=$ tiempo mediano, correspondiente al paso del $50 \%$ de la cantidad del trazador $=6.7 \mathrm{~h}$ y 10.2 \\
$\mathrm{~h}$ para cada fase respectivamente. \\
$\mathrm{t}_{\mathrm{f}}=$ tiempo que transcurre hasta que atraviesa la totalidad del trazador $=21.8 \mathrm{~h}$ y 28 h respectiv- \\
amente \\
$\mathrm{e}=$ excentricidad $=\{(\mathrm{t}$-tp)-(tp-ti) $\} /$ to
\end{tabular}

Tabla 2. Análisis de las curvas de tendencia del trazador

*Fuente: Adaptado de CEPIS (2004); Pérez y Torres (2008)

Se encontró que en las dos fases del estudio, el reactor presentó un predominio de flujo arbitrario o dual y mezcla completa, existiendo algo de cortos circuitos. La presencia de zonas muertas no es muy evidente ya que el valor de " $\mathrm{t}_{\mathrm{m}}$ " fue menor a " $\mathrm{t}_{\mathrm{o}}$ " en ambos ensayos. Sin embargo, teniendo en cuenta la recomendación de duración del ensayo ( 3 veces el valor de " $t$ ”") y los valores de " $t_{f}$ " de ambas fases, se observó que en la primera fase el trazador tardó más tiempo en salir del sistema que en el segundo ensayo lo que se ratifica con un mayor valor del cociente $t_{m} / t_{0}$ para el primer ensayo.

En general, podría decirse que no se observaron zonas de recirculación interna que pudieran retener el trazador en el reactor (Rojas y García, 2010) y que de acuerdo con lo mencionado por van Haandel y Lettinga (1994) en el reactor UASB predomina más el patrón de flujo de mezcla completa que el de flujo pistón; así mismo, como lo indica el modelo de Bolle et al (1986), el patrón de mezcla completa predomina a lo largo del manto de lodos y solo en el clarificador tiende a flujo a pistón.

Se destaca que la variación del caudal a lo largo del ensayo, probablemente influyó en la señal de salida del trazador. Por lo tanto es recomendable realizar posteriores estudios que abarquen los diferentes periodos de la curva de variación horaria de descargas de agua residual y compararlos con el régimen hidráulico predominante para dicha condición. El estudio de Peña et al (2006), demostró que el reactor UASB operando al TRH de diseño tiende a tener un patrón de flujo predominante de mezcla completa. Valores de TRH inferiores o superiores a dicha condición, generan disturbios hidráulicos en el reactor que ocasionan el predominio de patrones de flujo dual o arbitrario.

\section{Cálculo de la curva $\mathrm{E}(\mathrm{t})$ y aplicación de modelo de dispersión axial}

La Figura 4 esquematiza la curva de distribución de tiempos $\mathrm{E}(\mathrm{t})$, a partir de la cual se determinó el Tiempo de Retención Hidráulico experimental o real obtenido en cada ensayo mediante la integral de $\mathrm{t}^{*} \mathrm{E}(\mathrm{t})$. 
Se observa en las curvas $\mathrm{E}(\mathrm{t})$ que los valores del TRH teórico y experimental presentaron valores muy cercanos en la primera fase, a diferencia de la Fase 2 en la cual se presentó un TRH real muy inferior al teórico. Esto se puede relacionar con la presencia de cortos circuitos o líneas de flujo preferencial, lo que se ratifica con el análisis de la curva de concentración del trazador (Tabla 2) en la que la fracción $t_{i} / t_{0}<0.3$ fue más baja para la segunda fase. Comparando los resultados obtenidos con los reportados por Peña et al (2006), quienes evaluaron un reactor UASB con diferentes TRH (entre 10.6 y 4.9 horas), se observa que los TRH experimentales también fueron inferiores a los teóricos (entre 7.0 y 4.3 horas) siendo más evidente la diferencia con los TRH más elevados.
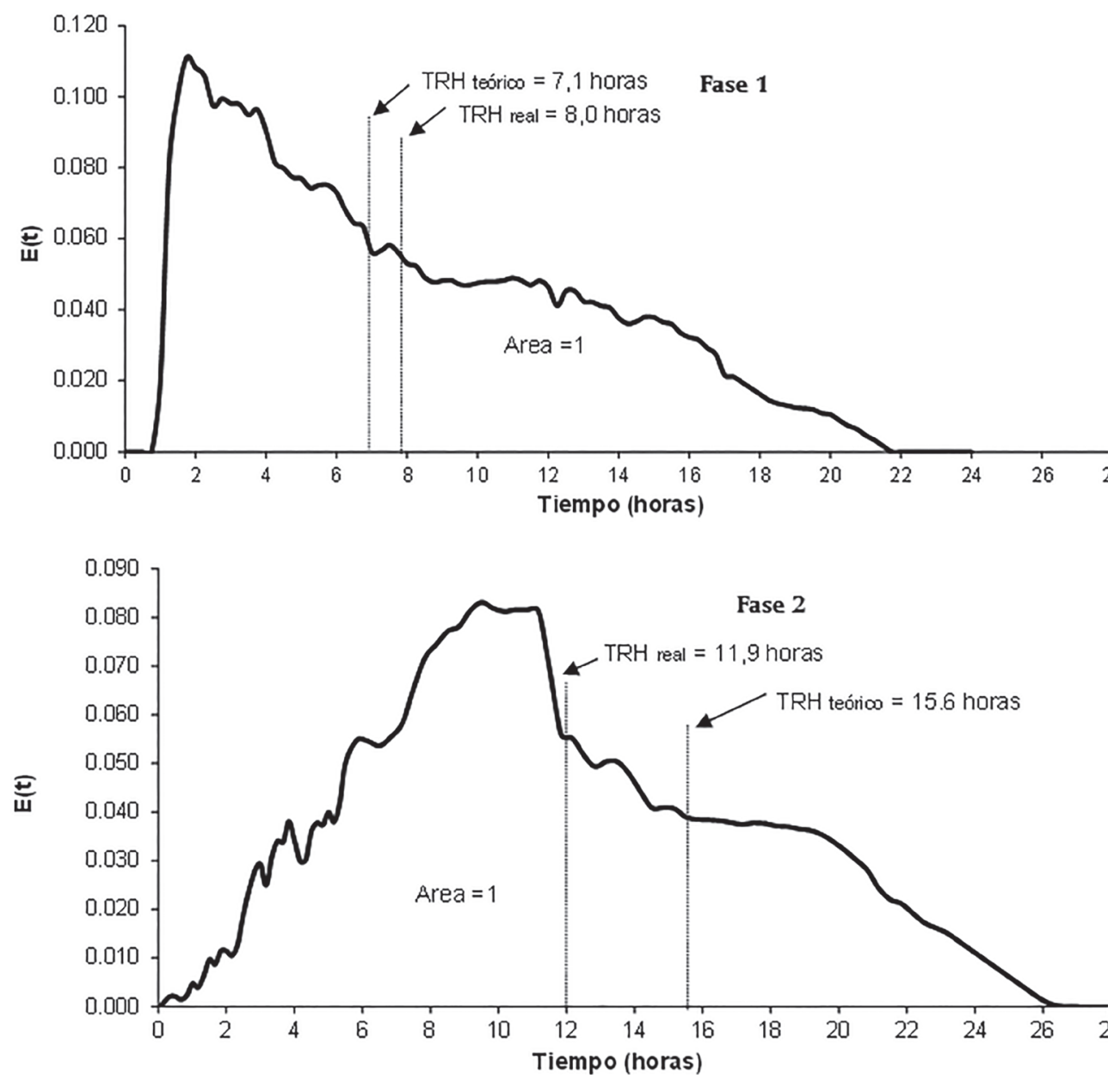

Figura 4. Curvas de E(t) vs tiempo. 
Aplicando el modelo dispersión axial (Levenspiel, 1997) se encontró un valor del índice d/uL de 0.20 para la Fase 1 y 0.14 para la Fase 2, lo que muestra que la fracción de flujo a pistón en el reactor fue mayor en la fase $1 \mathrm{y}$ un predominio de mezcla completa en la Fase 2.El comportamiento obtenido en este estudio es similar al reportado por Peña et al., (2006) quienes reportan valores del índice de dispersión entre 0.145 y 0.720 , valores que fueron incrementándose a medida que el TRH disminuía.

\section{Aplicación de los modelos de Wolf-Resnick y Reactores Completamente Mezclados (RCM) en Serie}

La Tabla 3 sintetiza los valores obtenidos para cada fracción de flujo obtenidos a partir de la aplicación del modelo matemático de Wolf - Resnick (1963). Se destaca la ausencia de zonas muertas y predominio el flujo de Mezcla Completa ( $>50 \%$ ) en el reactor, siendo más evidente este patrón de flujo bajo las condiciones operacionales de la Fase 1. Estos resultados están en concordancia con lo reportado en el estudio de Haskoning (1989) citado por Van Haandel y Lettinga (1996), en el cual se evaluó un reactor UASB al inicio y un mes después de iniciado el arranque.

\begin{tabular}{|c|c|c|}
\hline PARÁMETRO & FASE 1 & FASE 2 \\
\hline$\Theta$ & 0,25 & 0,6 \\
\hline Tan $\alpha$ & 0,53191 & 0,50000 \\
\hline \% Pistón & $23.4 \%$ & $40.8 \%$ \\
\hline \% Zonas muertas & 0 & 0 \\
\hline \%Mezcla completa & $76.6 \%$ & $59.2 \%$ \\
\hline
\end{tabular}

Tabla 3. Resultados del modelo de Wolf-Resnick en las dos fases del estudio

La Figura 5 presenta los resultados de la aplicación del modelo de RCM en serie, resaltándose que en la Fase 1 el reactor UASB se aproximó a la curva de un solo Reactor Completamente Mezclado, lo que a su vez coincide con lo encontrado en el modelo de Wolf Resnick (76.6\% mezcla completa). Se observa que en la Fase 2 el predominio de la fracción de flujo a pistón que mostró el modelo de Wolf Resnick (40.8\%) también se reflejó en su comportamiento como un RCM en serie de 5. 

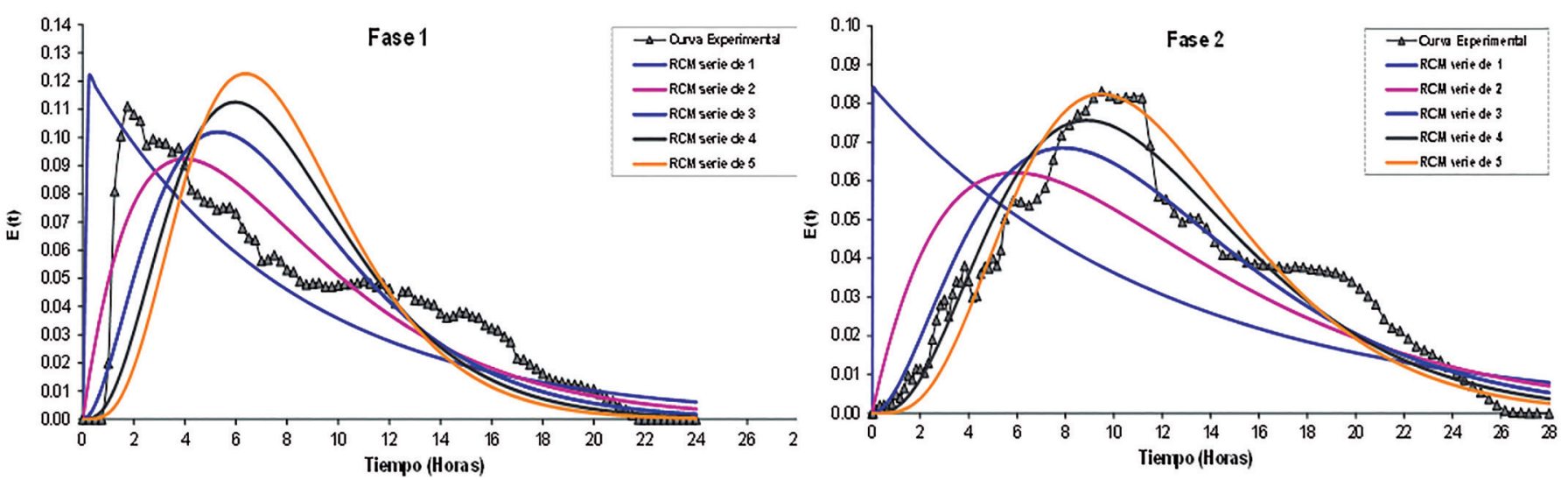

Figura 5. Curvas de E(t) vs tiempo del modelo de RCM en serie.

Los resultados de este estudio mostraron que aunque la formación del manto de lodos influyó en el comportamiento hidrodinámico en el reactor, la variación del TRH es un factor clave en el patrón de flujo predominante en el reactor, como también lo indicó el trabajo de Peña et al (2006). Estos autores afirmaron que en condiciones de baja carga hidráulica, hay un patrón de flujo hidrodinámicamente disperso con zonas de mezcla completa, zonas muertas y cortos circuitos, concentradas principalmente en el fondo del reactor y el manto de lodos. Bajo la condición del TRH de diseño el reactor se aproxima a un patrón de flujo ideal de mezcla completa; sin embargo, independiente si el reactor opera sobrecargado o con cargas inferiores a las de diseño, ambas condiciones ocasionan distorsiones en el flujo de mezcla completa ocasionando el predominio de flujo arbitrario.

\section{CONCLUSIONES}

El reactor UASB presentó una combinación entre flujo a pistón y mezcla completa independiente de la presencia o no del manto de lodos, siendo predominante el flujo mezclado al inicio del arranque (76.6\%) comparado con la fase final (59.2\%). Se destaca que en ninguna de las dos fases evaluadas se encontró presencia de zonas muertas.

La mayor proporción de flujo a pistón evidenciada al final del arranque se ratificó con el modelo de reactores completamente mezclados en serie, con el cual se observó un incremento en el número de reactores de 1 a 5 reactores en serie para las Fases 1 y 2 respectivamente. 
El número de dispersión axial disminuyó en la Fase 2 reconfirmando el incremento de la fracción de flujo pistón. Sin embargo, se destaca que las experiencias reportadas en la literatura indican que a medida que se incrementa el TRH disminuye el valor del índice de dispersión, situación que se presentó en el estudio en el cual, la fase 1 operó con un TRH experimental menor (8 horas) comparada con la Fase 2 (11.9 horas).

Aunque la formación del manto de lodos influyó en el comportamiento hidrodinámico en el reactor, la variación del TRH, reflejado en la curva de variación del caudal, es un factor clave en el patrón de flujo predominante en el reactor por influir en la señal de salida del trazador. Para ensayos en reactores operando a escala real sin caudal constante, se recomienda realizar estudios adicionales que abarquen los diferentes periodos de la curva de variación horaria de descargas de agua residual.

\section{REFERENCIAS BIBLIOGRÁFICAS}

Álvarez, J, A., Ruiz, I., Gómez, M., Presas, J., \& Soto, M. (2006). Start-up alternatives and performance of an UASB pilot plant treating dilutes municipal wastewater at low temperatures, Bioresource Technology, 97, 1640-1649.

Arroyave, D., González, M., Gallego, D. (2005). Evaluación del comportamiento hidráulico un reactor UASB utilizado para el tratamiento de aguas residuales. XXI Congreso Interamericano de Ingeniería Química. VIII Congreso Peruano de Ingeniería Química. Lima Perú. Disponible en: http://www. ciiq.org/varios/peru_2005/Trabajos/II/3/3.3.11.pdf

Avella, G. (2001). Evaluación del comportamiento hidrodinámico de un reactor UASB y su influencia en la remoción de materia orgánica. Cali. Tesis de grado (M.Sc. Ingeniería Sanitaria y Ambiental). Universidad del Valle. Facultad de Ingeniería. Postgrado en Ingeniería Sanitaria y Ambiental.

Bhattacharyya, D. and Singh, K. (2010). Understanding the Mixing Pattern in an Anaerobic Expanded Granular Sludge Bed Reactor: Effect of Liquid Recirculation. Journal of Environmental Engineering, 136(6), 576-584.Online publication date: 1-Jun-2010

Bolle, W, L., van Breugel, J., van Eybergen, G, C., Kossen, N, W, F., \& Zoetemeyer, R, J. (1986). Modeling the liquid flow in up-flow anaerobic sludge blanket reactors. Biotechnology and Bioengineering, 28, 1615-1620.

Bolle, W, L., van Breugel, J., van Eybergen, G, C., Kossen, N, W, F., \& van Gils, W. (1986). An integral dynamic model for the UASB reactor. Biotechnology and Bioengineering, 28, 1621-1636.

Centro Panamericano de Ingeniería Sanitaria y Ambiental - CEPIS. Tratamiento de agua para consumo humano. Filtración Rápida. Perú. 2004. 
Chernicharo, C, A. (1997). Reatores anaerobios. Principios do tratamento biológico de aguas residuarias. Vol.5. Universidade Federal de Minas Gerais

Chowdhury, R. and Mehrotra, I. (2004). Minimization of Short-Circuiting Flow through Upflow Anaerobic Sludge Blanket Reactor. Journal of Environmental Engineering. 130 (9), 951-959. Technical Papers.

Galvis, G. (2005). Análisis de flujos y factores que determinan los periodos de retención, tomo II. Manual No 4. Programa Regional HPE/OPS/CEPIS de Mejoramiento de la Calidad del Agua para Consumo Humano. Lima, CEPIS/OPS.

Giácoman, G. Rejón, L.I. Aguilar, S.R. (2006). Evaluación de las características hidráulicas de un reactor de lecho fluidificado para el tratamiento aerobio de las aguas residuales". México. XV Congreso Nacional de Ingeniería Sanitaria y Ciencias Ambientales.

Grady, L., Daigger, G., Love, N., Filipe, C, D, M. (2011). Biological wastewater treatment. 3th Ed, IWA, CRP Press, Boca Raton.

Hulshoff L, Lettinga G, (1988). Process design for varios type of waste water. Departament of Enviromental Technology. Wageningen Agricultural University

Hulshoff L, Lettinga G, (1988). Process design for varios type of waste water. Departament of EnviromentalTechnology. Wageningen Agricultural University. 6. Hulshoff. Citado por Arroyave et al (2005).

Iñiguez-Covarrubias G., Camacho-López A. (2011). Evaluación de un reactor de manto de lodo con flujo ascendente (UASB) con cambios de velocidad de alimentación. Ingeniería Investigación y Tecnología. Vol. XII, Núm. 1, 199-208

Jiménez, B., Noyola, A., \& Capdeville, B. (1988). Selected dyes for residence time distribution evaluation in bioreactors. Biotechnology Techniques, 2 (2), 77-82.

Lettinga, G., van Velsen, A, F, M., Hobma, S, W., de Zeeuw, W., and Klapwijk, A. (1980). Use of the upflow sludge blanket (USB) reactor concept for biological wastewater treatment, especially for anaerobic treatment, Biotechnology and Bioengineering, 22 (4), 699-734.

López, I. and Borzacconi, L. (2010). UASB reactor hydrodynamics: residence time distribution and proposed modelling tools. Environmental Technology, 31(6), 591-600. Online publication date: 1-May-2010.

Lema, J, M., Méndez, R., Iza, J., García, P., \& Fernández-Polanco, F. (1991). Chemical reactor engineering concepts in design and operation of anaerobic treatment processes. WaterScience and Technology, 24 (8), 79-86. 
Levenspiel, O. (1997). Ingeniería de las reacciones químicas. Departamento de Ingeniería Química de la Universidad de Oregon. Ed. Reverté, S.A. Jhon Wiley and Sons. New York, 638 p.

M.A. Rocha, H. Normando, C. Onofre. (2000). Aplição de tracadores em filtros anaeróbicos para avaliação das características hidrodinâmicas. Porto Alegre, Brasil. Associação Brasileira de Engenharia Sanitária e Ambiental - Região Rio Grande do Sul (ABES/RS). XXVII Congresso Interamericano de Engenharia Sanitária e Ambiental.

Morgan- Sagastume, J, M., Jiménez, B., \& Noyola, A. (1997). Tracer studies in a laboratory and pilot scale UASB reactor. Environmental Technology, 18, 817-825.

Nnaji, C. (2013). A review of the upflow anaerobic sludge blanket reactor. Desalination and Water Treatment, 1-22. Online publication date: 4-Jun-2013

Peña, M, R., Mara, D., Avella, G. (2006). Dispersion and treatment performance analysis of an UASB reactor under different hydraulic loading rates. Water Research, 40 (3), 445-452.

PTAR "Río Frío" exitosa aplicación de la tecnología UASB, a escala real, para el tratamiento de las aguas residuales domésticas en Bucaramanga (Colombia) / C.J.Collazos y J.M.Cala. Presentado al Segundo Taller Regional Tratamiento Anaerobio de Aguas Residuales en América Latina. Ciudad de la Habana, http://www.bvsde.ops-oms.org/eswww/fulltext/repind54/ptar/ptar.html

Rebhum. M., \& Argaman Y. (1965). Evaluation and hydraulic efficiency of sedimentations basins. Journal Sanitary Engineering Division, 91, 37-45.

Rojas, A., Garcia, A. (2010). Análisis de la curva de distribución del tiempo de residencia en un sistema de lixiviación. Tecnología Química, 15 (1), 61- 68.

Schellinkhout, A., \& Collazos, C, J. (1992). Full-Scale applications of the UASB technology for sewage treatment. Water Science and Technology, 25 (7), 159-166.

Singh, K.,Viraraghavan, T., and Bhattacharyya, D.(2006). Sludge Blanket Height and Flow Pattern in UASB Reactors: Temperature Effects. Journal of Environmental Engineering.,132 (8), 895-900.

Van Haandel, A., Lettinga, G. (1994). Anaerobic sewage treatment. Wiley. New York

Von Sperling, M.(1996). Principios básicos do tratamento de esgotos. Vol 2. Principios do tratamento biologico de aguas residuarias. DESA, UFMG, Brasil, 210p.

Weber. W. \& DiGiano.F. (1996). Process dynamics in environmental systems. New York, NY: Wiley Interscience. 
Wolf, D. y Resnick, N. (1963). Residence time distribution in real systems.I.E.C. Fundamentals, 2(4): 287. Citado por Galvis, G. (1984). Consideraciones sobre la aplicación del modelo simplificado de Wolf y Resnick. XXVII congreso nacional de Ingeniería Sanitaria y Ambiental. Barranquilla.

Zeng, Y., Mu, S, J., Lou, S. J., Tartakovsky, B., Guiot, S, R., y Wu, P. (2005). Hydraulic modeling and axial dispersion analysis of UASB reactor. Biochemical Engineering Journal, 25, 113- 123. 